\title{
Fractional Diffusion Equation Approach to the Anomalous Diffusion on Fractal Lattices
}

\author{
Dann Huh, Jinuk Lee, and Sangyoub Lee* \\ Department of Chemistry, Seoul National University, Seoul 151-747, Korea. *E-mail: sangyoub@snu.ac.kr \\ Received August 26, 2005
}

\begin{abstract}
A generalized fractional diffusion equation (FDE) is presented, which describes the time-evolution of the spatial distribution of a particle performing continuous time random walk (CTRW) on a fractal lattice. For a case corresponding to the CTRW with waiting time distribution that behaves as $\psi(t) \sim t^{-(\alpha+1)}$, the FDE is solved to give analytic expressions for the Green's function and the mean squared displacement (MSD). In agreement with the previous work of Blumen et al. [Phys. Rev. Lett. 1984, 53, 1301], the time-dependence of MSD is found to be given as $\left\langle r^{2}(t)\right\rangle \sim t^{2 \alpha / d_{w}}$, where $d_{w}$ is the walk dimension of the given fractal. A Monte-Carlo simulation is also performed to evaluate the range of applicability of the proposed FDE.
\end{abstract}

Key Words : Fractional diffusion equation, Continuous time random walk, Dispersive diffusion, Sierpinski gasket, Percolation cluster

\section{Introduction}

Anomalous diffusion is frequently observed in disordered media. It is characterized by non-linear time dependence of the mean squared displacement (MSD),

$$
\left\langle r^{2}(t)\right\rangle \sim t^{\gamma}
$$

with $\gamma \neq 1$. For the case with $0<\gamma<1$, the process is called subdiffusive or dispersive and such an anomaly is found in many physical systems including glasses, amorphous semiconductors, lipid bilayers, and living cells. ${ }^{1-5}$

One of the representative theories proposed to explain the anomalous diffusion phenomena is that based on the continuous time random walk (CTRW) model. ${ }^{2,6}$ In this model the random walker makes a jump between lattice points after a certain waiting time that is chosen randomly from a distribution function $\psi(t)$. For a power-law waiting time distribution $\psi(t) \sim t^{-(1+\alpha)}$ with $0<\alpha<1$, the first moment of $\psi(t)$ diverges and the MSD is given by $\left\langle r^{2}(t)\right\rangle \sim t^{\alpha}$. Such a subdiffusive CTRW occurring on the regular lattice without spatial constraints has been shown to be well described by the fractional diffusion equation (FDE) or the fractional Fokker-Planck Equation (FFPE) for intermediate to long time regime. ${ }^{7-10}$ The utility of a fractional kinetic equation approach rests on the availability of an analytic expression for the Green's function, in terms of which many dynamical quantities can be evaluated in a straightforward manner. ${ }^{7}$

Another source of subdiffusion is the spatial constraint posed by heterogeneous molecular environments in disordered media. The fractals are often considered as a model of such systems. ${ }^{1,11-14}$ For a particle moving along the fractal lattice with a fixed jump frequency, the MSD is known to be given by $\left\langle r^{2}(t)\right\rangle \sim t^{2 / d_{w}}$ where $d_{w}$ is the walk dimension with $d_{w}>2$ in most cases. ${ }^{11,12}$ For the case of regular lattices devoid of connectivity defects, $d_{w}$ is equal to 2 so that the result for normal Fickian diffusion is recovered. To explain this and other diffusion-related properties associated with the fractal lattices, several diffusion equations have been proposed with the respective Green's functions. ${ }^{15-18}$

For the case involving both origins of subdiffusion - the temporal constraint and the spatial constraint - Blumen et al. applied the CTRW formalism to the fractal lattice and derived the expression for $\gamma$ in Eq. (1). ${ }^{14}$ The idea was to separate the temporal and the spatial parts of MSD and link them as

$$
\left\langle r^{2}(t)\right\rangle=\sum_{n=0}\left\langle r_{n}^{2}\right\rangle \chi_{n}(t)
$$

where $\left\langle r_{n}^{2}\right\rangle$ is the average distance traveled in $n$ steps and $\chi_{n}(t)$ is the probability of having performed just $n$ steps by $t$. It was shown that the time exponent is given by

$$
\gamma=2 \alpha / d_{w}
$$

In this work we present a generalized fractional diffusion equation (FDE), which describes the time-evolution of the spatial distribution of a particle performing CTRW on a fractal lattice. The proposed FDE is solved to give analytic expressions for the Green's function and the MSD. Although this approach provides expressions for the particle distribution and the MSD for all times, the range of applicability of these expressions needs to be examined. We therefore carry out Monte-Carlo simulations of CTRW on the Sierpinski gasket and the percolation cluster. For both fractal lattices, the MSD expression obtained from the proposed FDE is in good agreement with the simulation result from intermediate to long times. However, the particle displacement distribution function is in agreement with the simulation result only for the Sierpinski gasket case. For the percolation cluster case, the simulation result displays a little more dispersed distribution than that predicted by the proposed FDE. 


\section{Generalized Fractional Diffusion Equation}

We consider a particle which performs CTRW on a fractal lattice with the waiting time distribution $\psi(t)$. If the spatial and the temporal constraints are uncorrelated, the generalized master equation describing the CTRW process can be reduced in the continuum limit to the generalized fractional diffusion equation. ${ }^{7,9}$ In Laplace domain, it can be written as

$$
s \hat{p}(r, s)-p(r, 0)=\frac{s \hat{\psi}(s)}{1-\hat{\psi}(s)} L(r) \hat{p}(r, s),
$$

where $p(r, t)$ is the average probability density of finding the particle at a position located at the radial distance $r$ at time $t$, and $\hat{f}(s)$ denotes the Laplace transform of $f(t) . L(r)$ is the diffusion operator for a particle moving on the given fractal lattice with a fixed jump frequency. O'Shaughnessy and Procaccia ${ }^{15}$ obtained an expression for $L(r)$ on the basis of a scaling argument with the introduction of a distancedependent diffusion kernel. The expression is given by

$$
L(r)=\frac{D_{s p}}{r_{f}-1} \frac{\partial}{\partial r}\left(\frac{r^{d_{f}-1}}{r^{d_{w}-2}} \frac{\partial}{\partial r}\right)
$$

where $d_{f}$ is the fractal dimension. Although it is known that this diffusion operator may give inaccurate results when $r / t^{1 / d_{w}}$ is large, ${ }^{16-19}$ it will be used here to get an analytic solution to Eq. (4). For regular lattices, $D_{s p}=l^{2} / 2 D$ where $l$ is the spacing between adjacent lattice sites and $D$ is the dimension of the Euclidean space embedding the lattice. For the case of Sierpinski gasket, O'Shaughnessy and Procaccia ${ }^{15}$ obtained an expression for $D_{s p}$ that is given by

$$
D_{s p}=\frac{l^{d_{w}}}{(D+1) d_{f}\left(d_{w}-d_{f}\right)} .
$$

An interesting model for dispersive transport in amorphous semiconductors was presented by Jakobs and Kehr, ${ }^{20}$ which leads to a power-law waiting time distribution. In this model, the release of the particle from a lattice site is assumed to be an activated process. The release rate is given by

$$
v_{r}(E)=v_{0} \exp \left(-E / k_{B} T\right)
$$

where $E$ is the energy barrier at a given site. $k_{B}$ is the Boltzmann constant and $T$ is the absolute temperature. Then the energy barriers are assumed to be distributed according to an exponential distribution,

$$
f(E)=\frac{1}{k_{B} T_{c}} \exp \left(-E / k_{B} T_{c}\right)
$$

where $k_{B} T_{c}$ is a characteristic energy defining the distribution. If we suppose that the particle is trapped at a lattice site at time zero, then the probability that it is released from the site at time $t$ is given by

$$
\psi(t)=\int_{0}^{\infty} d E f(E) v_{r}(E) \exp \left(-v_{r}(E) t\right)
$$

$$
=\frac{\alpha v_{0} \gamma\left(\alpha+1, v_{0} t\right)}{\left(v_{0} t\right)^{\alpha+1}}
$$

where $\alpha \equiv T / T_{c}$ and $\gamma$ is the incomplete gamma function. ${ }^{21}$ For $v_{0} t>>1$, the waiting time distribution $\psi(t)$ in Eq. (9) becomes

$$
\psi(t) \cong \frac{\alpha v_{0} \Gamma(\alpha+1)}{\left(v_{0} t\right)^{\alpha+1}} .
$$

The Laplace transform of the waiting time distribution $\psi(t)$ in Eq. (9) can be expressed in terms of the hypergeometric functions, ${ }^{22}$ but its use in Eq. (4) leads to too complicated equation. We will thus use the leading term approximation for $\hat{\psi}(s)$ in the small $s$ limit,

$$
\hat{\psi}(s) \cong 1-\frac{\pi \alpha}{\sin \pi \alpha}\left(\frac{s}{v_{0}}\right)^{\alpha} .
$$

Substitution of Eqs. (5) and (11) into Eq. (4) yields

$$
s \hat{p}(r, s)-p(r, 0)=D_{\alpha f} \frac{s^{1-\alpha}}{r^{d_{f}-1}} \frac{\partial}{\partial r}\left(\frac{r^{d_{f}-1}}{r^{d_{w}-2}} \frac{\partial}{\partial r}\right) \hat{p}(r, s) .
$$

Here, the generalized diffusion coefficient $D_{\alpha f}$ is given by

$$
D_{\alpha f}=\frac{\sin \pi \alpha}{\pi \alpha} v_{0}^{\alpha} D_{s p}
$$

For the CTRW on regular lattices, this expression reduces to that obtained by Barkai et al. in Ref. 9 .

Using the fractional differential operator

$$
{ }_{0} D_{t}^{1-\alpha} f(t) \equiv \frac{1}{\Gamma(\alpha)} \frac{\partial}{\partial t} \int_{0}^{t} d \tau \frac{f(\tau)}{(t-\tau)^{1-\alpha}} \text { for } 0<\alpha<1
$$

we can convert Eq. (12) back into time domain as

$$
\frac{\partial p(r, t)}{\partial t}={ }_{0} D_{t}^{1-\alpha}\left[\frac{D_{\alpha f}}{r_{f}-1} \frac{\partial}{\partial r}\left(\frac{r^{d_{f}-1}}{r^{d_{w}-2}} \frac{\partial}{\partial r}\right) p(r, t)\right] .
$$

Equation (14) is the generalized fractional diffusion equation for describing the particle transport in disordered fractal network.

\section{Green's Function and MSD}

To solve Eq. (14), we set the substitution variables as $\beta=$ $d_{w} / 2, d_{s}=d_{f} / \beta$, and $y=r^{\beta} / \beta$. Using these variables, Eq. (14) can be rewritten as

$$
\frac{\partial p(y, t)}{\partial t}={ }_{0} D_{t}^{1-\alpha}\left[\frac{D_{\alpha f}}{d^{d_{s}-1}} \frac{\partial}{\partial y}\left(y^{d_{s}-1} \frac{\partial}{\partial y}\right) p(y, t)\right] .
$$

In this equation, we see that the spatial differential operator has the familiar form of radial Laplacian, although the fracton dimension $d_{s}$ is not an integer in general. Hence we can expect that the solution of Eq. (15) may be represented in terms of the Bessel functions in Laplace domain. With the initial and the boundary conditions given by $p(r, t=0)=\delta(r)$, 
$\lim _{r \rightarrow \infty} \hat{p}(r, s)=0$, and $\left.(\partial / \partial r) \hat{p}(r, s)\right|_{r=0}=0$, it can be shown that the Green's function is given by

$$
\begin{aligned}
\hat{p}(y, s \mid 0) & =\frac{1}{\gamma_{d f} 2^{v} \Gamma(v+1) D_{\alpha f}^{1 / \alpha} \beta^{2 v+1}} \\
& \times \frac{K_{v}\left(\lambda^{\alpha / 2} y\right)}{y^{v}} \lambda^{\alpha(v / 2+1)-1}
\end{aligned}
$$

where $\lambda \equiv s / D_{\alpha f}^{1 / \alpha}$ and $v \equiv d_{s} / 2-1 . K_{v}(x)$ is the Bessel function of the second kind, and $\gamma_{d f}=2 \pi^{d_{f} / 2} / \Gamma\left(d_{f} / 2\right)$ is the metric factor.

The expression for the Green's function in time domain can be obtained from Eq. (16) as

$$
\begin{aligned}
p(r, t \mid 0) & =\frac{\beta}{\gamma_{d f} \Gamma\left(d_{s} / 2\right) r^{d_{f}}} \\
& \times H_{12}^{20}\left(\frac{r^{\beta}}{2 \beta} \frac{1}{\sqrt{D_{\alpha f} t^{\alpha}}} \mid \begin{array}{l}
(1, \alpha / 2) \\
(1,1 / 2) \quad\left(d_{s} / 2,1 / 2\right)
\end{array}\right),
\end{aligned}
$$

where $H_{p q}^{m n}$ is the Fox $H$-function defined as the MellinBarnes type path integral ${ }^{10}$

$$
\begin{aligned}
H_{p q}^{m n}\left(z \mid \begin{array}{l}
\left(a_{p}, A_{p}\right) \\
\left(b_{q}, B_{q}\right)
\end{array}\right) & =H_{p q}^{m n}\left(z \mid \begin{array}{l}
\left(a_{1}, A_{1}\right),\left(a_{2}, A_{2}\right), \ldots,\left(a_{p}, A_{p}\right) \\
\left(b_{1}, B_{1}\right),\left(b_{2}, B_{2}\right), \ldots,\left(b_{q}, B_{q}\right)
\end{array}\right) \\
& =\frac{1}{2 \pi i} \int_{L} d s \chi(s) z^{s}
\end{aligned}
$$

with the integral density

$$
\chi(s)=\frac{\prod_{1}^{m} \Gamma\left(b_{j}-B_{j} s\right) \prod_{1}^{n} \Gamma\left(1-a_{j}+A_{j} s\right)}{\prod_{m+1}^{q} \Gamma\left(1-b_{j}+B_{j} s\right) \prod_{n+1}^{p} \Gamma\left(a_{j}-A_{j} s\right)} .
$$

By using the property

$$
H_{p q}^{m n}\left(x \mid \begin{array}{c}
\left(a_{p}, A_{p}\right) \\
\left(b_{q}, B_{q}\right)
\end{array}\right)=k H_{p q}^{m n}\left(x^{k} \mid \begin{array}{c}
\left(a_{p}, k A_{p}\right) \\
\left(b_{q}, k B_{q}\right)
\end{array}\right),
$$

it can be shown easily that for the case of subdiffusion occurring on the regular lattces with $\beta=1$, Eq. (17) reduces to Eq. (29) of Ref. 9:

$$
p(r, t \mid 0)=\frac{1}{\alpha \pi^{D / 2} r^{D}} H_{12}^{20}\left(\left(\frac{r}{2}\right)^{2 / \alpha} \frac{1}{D_{\alpha f}^{1 / \alpha} t} \mid \begin{array}{l}
(1,1) \\
(1,1 / \alpha)(D / 2,1 / \alpha)
\end{array}\right) .
$$

On the other hand, when the waiting time distribution has a finite first moment $\left(e . g\right.$. $\psi(t)=\tau_{1}^{-1} e^{-t / \tau_{1}}$ or $\psi(t)=$ $\left.\delta\left(t-\tau_{1}\right)\right)$, Eq. (12) becomes

$$
s \hat{p}(r, s \mid 0)-\delta(r)=\left(\tau_{1}^{-1} D_{s p}\right) \frac{1}{r^{d_{f}-1}} \frac{\partial}{\partial r}\left(\frac{r^{d_{f}-1}}{r^{d_{w}-2}} \frac{\partial}{\partial r}\right) \hat{p}(r, s \mid 0) .
$$

Hence, by setting $\alpha=1$ and $D_{\alpha f}=\tau_{1}^{-1} D_{s p}$ in Eq. (17) and by using the property $H_{01}^{10}\left(x \mid \frac{}{(b, 1)}\right)=x^{b} e^{-x}$, we can show that Eq. (17) reduces to Eq. (5) of Ref. 15:

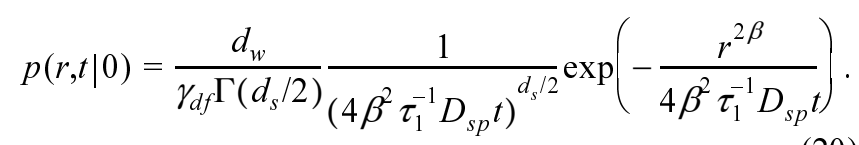

The MSD expression can be obtained more easily from inverse Laplace transformation of the spatial integral of Eq. (16), $\quad \int_{0}^{\infty} d r \gamma_{d f} r_{f}-1 r^{2} \hat{p}(r, s \mid 0)$, rather than by direct integration of Eq. (17). We have

$$
\left\langle r^{2}(t)\right\rangle=\frac{\Gamma\left(\left(d_{s} / 2\right)+(1 / \beta)\right) \Gamma(1+(1 / \beta))}{\Gamma\left(d_{s} / 2\right) \Gamma(1+(\alpha / \beta))}\left(4 \beta^{2} D_{\alpha f}\right)^{1 / \beta} t^{\alpha / \beta}
$$

This result is in agreement with that obtained by Blumen et $a l .{ }^{14} ;\left\langle r^{2}\right\rangle \sim t^{\alpha / \beta}$. If we set $\beta=1$, Eq. (21) reduces to Eq. (28) of Ref. 9 obtained for the case of subdiffusion occurring on the regular lattces.

\section{Comparison with Computer Simulation Results}

To derive the FDE presented in Eq. (12), we have invoked two approximations. The expressions for $L(r)$ in Eq. (5) and $\hat{\psi}(s)$ in Eq. (11) are valid for intermediate to long times. We therefore examine the range of applicability of the present theoretical results by comparison with the Monte-Carlo (MC) simulations.

We have simulated CTRW on the Sierpinski gasket, a deterministic fractal, and on the incipient percolation cluster, a random fractal. For each trajectory the initial position is chosen randomly. To generate the waiting times in conformity with the distribution in Eq. (9), we first generate the trap energy $E$ following the exponential distribution in Eq. (8) with $k_{B} T_{c}=10$. For this randomly chosen energy barrier, the value of the release rate $v_{r}(E)$ is calculated from Eq. (7) with $k_{B} T=\alpha k_{B} T_{c}$; for each fractal lattice, we carry out two sets of simulations with $\alpha=0.5$ and 0.7 . The waiting time is then obtained by multiplying $1 / v_{r}(E)$ to an exponentially distributed, positive, random deviate of unit mean. ${ }^{23}$
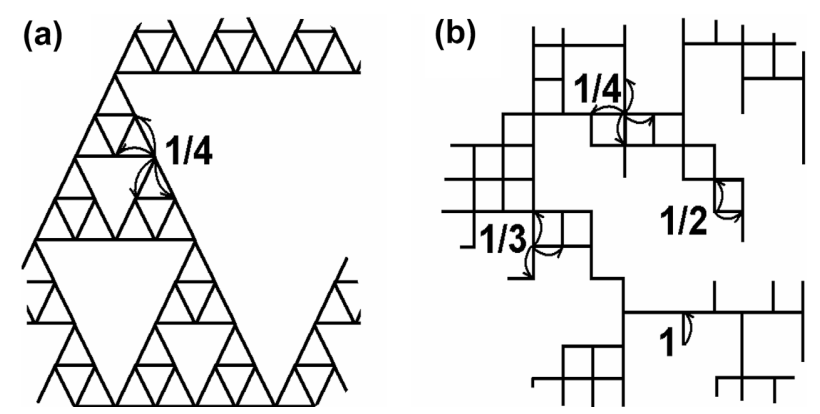

Figure 1. "Myopic ant" model for (a) Sierpinski gasket and (b) percolation cluster. 
For the CTRW on the Sierpinski gasket [see Fig. 1(a)], the particle may jump to any one of the four nearest neighboring sites with equal probability of $1 / 4$. After landing on a site at time $t$, the waiting time $\Delta t$ at the site is generated as described above and upon moving to the neighboring site, time advances to $t+\Delta t$. To avoid the system size effect, we use a very large lattice whose side length is $2^{22} l$. By comparing the simulation results with those obtained for the lattice with side length of $2^{21} l$, we have checked that the size effect is absent.

The 2-dimensional incipient percolation cluster as shown in Figure 1(b) is generated on the $2000 \times 2000$ square lattice. The sites are selected randomly with the probability of 0.5927 , and the CTRW is performed on the biggest cluster generated. Again, we have confirmed the absence of the system size effect by comparing simulation results with those obtained for a little smaller or larger lattice. The results appear to converge for the percolation clusters generated on square lattices larger than the $1500 \times 1500$ lattice. The initial position of the particle is randomly selected among the lattice sites located around the center of gravity of the whole cluster. In addition, for better statistics new clusters are generated for every 400 trajectories. The particle moves to the available nearest neighboring sites with equal probability, which is the reciprocal of the coordination number as shown in Figure 1(b). This move algorithm is called the 'myopic ant' model. ${ }^{12}$

In Figures 2 and 3, MC simulation results for MSD are compared with the analytical results from Eq. (21). We use the following reduced variables; $x=r / l$ and $\tau=t / t_{D}$ with $t_{D}=$ $\left(l^{\beta} / D_{\alpha f} \beta^{2}\right)^{1 / \alpha}$. The solid lines represent the analytic results, while the dotted curves are the simulation results obtained from 20,000 trajectories. In both figures, the upper curves are for the case with $\alpha=0.7$, while the lower curves for the case with $\alpha=0.5$.

For the CTRW on Sierpinski gasket (Fig. 2), the analytic results are in good agreement with simulations for times larger than 3. In this case, values of the fractal dimensional parameters are given as follows: $d_{f}=\ln 3 / \ln 2, d_{s}=\ln 9 / \ln 5$, and $\beta=d_{f} / d_{s .}{ }^{12}$ The generalized diffusion coefficient $D_{\alpha f}$ is

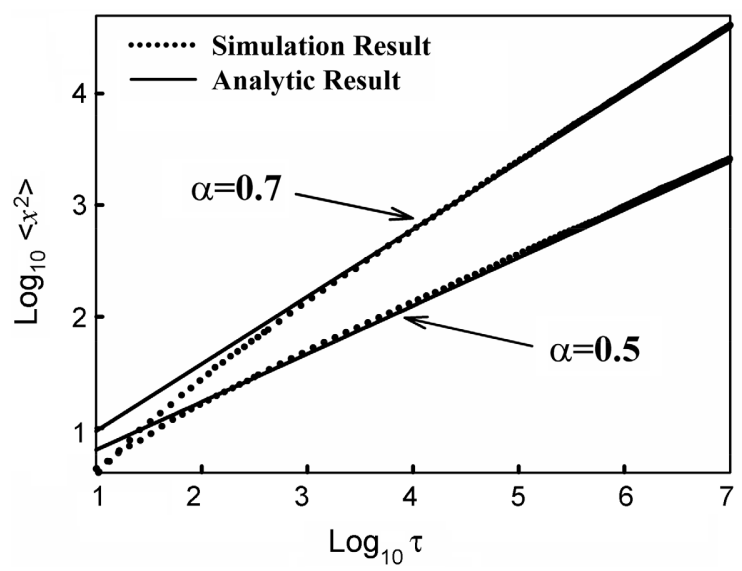

Figure 2. The mean squared displacement $\left\langle x^{2}\right\rangle$ as a function of time $\tau$ on Sierpinski gasket.

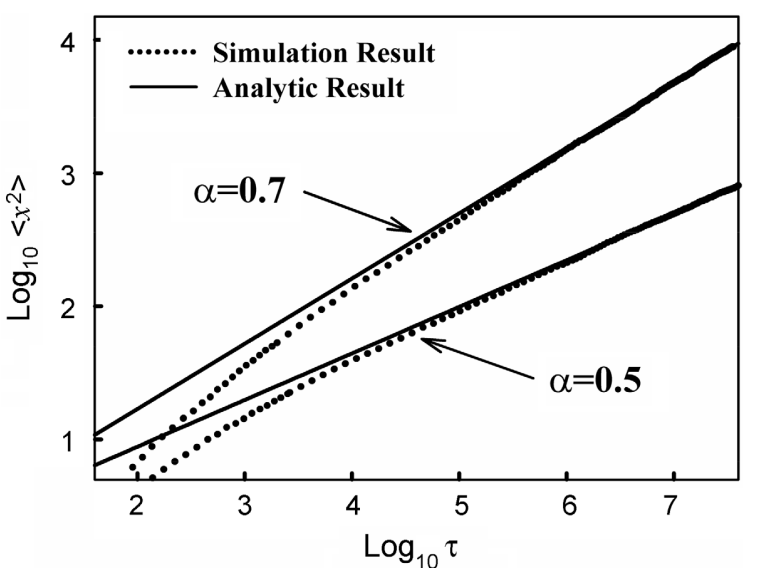

Figure 3. The mean squared displacement $\left\langle x^{2}\right\rangle$ as a function of time $\tau$ on incipient percolation cluster.

determined from Eqs. (6) and (13) without any adjustable variables. Hence the agreement between the MC simulations and Eq. (21) requires the correctness of the amplitude factor as well as the time exponent.

For the case of percolation cluster (Fig. 3), $d_{f}=91 / 48, d_{s}=$ 1.327 , and $\beta=d_{f} / d_{s},{ }^{12}$ but an analytic expression for $D_{s p}$ has not been given. To determine the value of $D_{s p}$, we perform independent simulations of a simple random walk with $\psi(t)$ $=\delta\left(t-\tau_{1}\right)$ on the percolation cluster. $D_{s p}$ can be determined by fitting the simulation results to the following equation,

$$
\left\langle y^{2}\right\rangle=2 d_{s} D_{s p}\left(t / \tau_{1}\right) \text { with } y=r^{\beta} / \beta
$$

which can be derived from Eq. (20). We find that $D_{s p}=$ 0.4775. This value is then substituted into Eq. (13) to calculate $D_{\alpha f}$. For the CTRW on percolation cluster, we see that MSD values calculated from the analytic expression in Eq. (21) are in agreement with simulations at longer times compared to the Sierpinski gasket case.

In Figures 4 and 5, MC simulation results for particle distribution $p(x, \tau) \gamma_{d f} x^{d_{f}-1}$ calculated from 70,000 trajectories are compared with the results obtained from the analytic expression in Eq. (17). The Fox $H$-function is

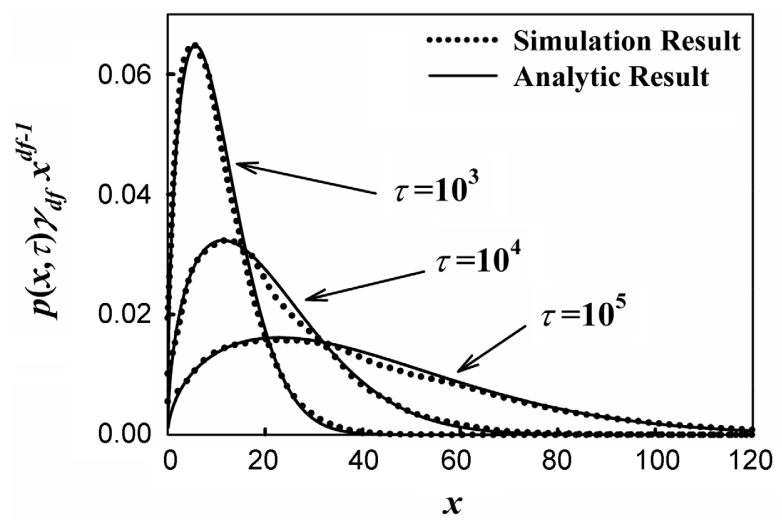

Figure 4. Particle distributions $p(x, \tau) \gamma_{d f} x^{d_{f}-1}$ for CTRW on Sierpinski gasket. The waiting time distribution exponent $\alpha$ is set to 0.7 . 


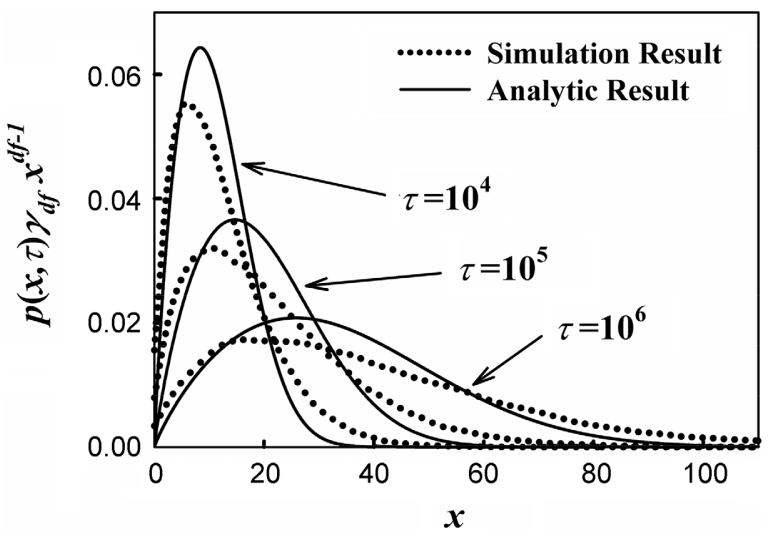

Figure 5. Particle distributions $p(x, \tau) \gamma_{d f} x^{d_{f}-1}$ for CTRW on incipient percolation cluster. The waiting time distribution exponent $\alpha$ is set to 0.7 .

evaluated by using the series expression, ${ }^{24}$

$$
\begin{gathered}
H_{p q}^{m n}(z)=\sum_{h=1}^{m} \sum_{v=0}^{\infty}\left[\frac{\prod_{j=1, j \neq h}^{m} \Gamma\left(b_{j}-B_{j}\left(b_{h}+v\right) / B_{h}\right)}{\prod_{j=m+1}^{q} \Gamma\left(1-b_{j}+B_{j}\left(b_{h}+v\right) / B_{h}\right)}\right. \\
\times \frac{\prod_{j=1}^{n} \Gamma\left(1-a_{j}+A_{j}\left(b_{h}+v\right) / B_{h}\right)}{\prod_{j=n+1}^{p} \Gamma(-1)^{v} z^{\left(b_{h}+v\right) / B_{h}}} \\
\left.v ! B_{h}\left(b_{h}+v\right) / B_{h}\right)
\end{gathered} .
$$

The plots are given for several values of $\tau$ with $\alpha=0.7$. For the case of Sierpinski gasket, the agreements between theory and simulations are excellent. However, for the case of incipient percolation cluster, the simulation results show that the particle distributions are more dispersed to longer distance than the analytical expression predicts.

\section{Concluding Remarks}

In this work we presented a generalized FDE for describing the particle transport in disordered media, which involve trapping sites with random energy barriers as well as spatial constraints. As a model we considered the CTRW on fractal lattices. Analytic expressions for Green's function of the FDE and the MSD were obtained. In agreement with the previous work, ${ }^{14}$ the time exponent appearing in the MSD expression was found to be $2 \alpha / d_{w}$, which reveals both origins of subdiffusion related to spatial and temporal constraints. Our approach provided an explicit expression for the generalized diffusion coefficient that determines the amplitude factor of MSD. Monte-Carlo simulations were also performed to evaluate the range of applicability of the proposed FDE. For the CTRW on Sierpinski gasket, the FDE provides an excellent description of the transport dynamics for intermediate to long times. However, in the percolation cluster case, only the MSD is reasonably reproduced by the FDE. The actual particle distribution observed in MC simulations deviates from that predicted by the FDE.

Acknowledgment. This work was supported by a grant from Korea Research Foundation (KRF-2004-015-C00234).

\section{References}

1. Blumen, A.; Klafter, J.; Zumofen, G. In Optical Spectroscopy of Glasses, Zschokke, I., Ed.; Reidel: Dordrecht, 1986; p 199.

2. Scher, H.; Montroll, E. W. Phys. Rev. B 1975, 12, 2455.

3. Wong, I. Y.; Gardel, M. L.; Reichman, D. R.; Weeks, E. R.; Valentine, M. T.; Bausch, A. R.; Weitz, D. A. Phys. Rev. Lett. 2004, 92, 178101 .

4. Ratto, T. V.; Longo, M. L. Langmuir 2003, 19, 1788.

5. Even, U.; Rademann, K.; Jortner, J. Phys. Rev. Lett. 1984, 52, 2164.

6. Montroll, E. W.; Weiss, G. H. J. Math. Phys. 1965, 6, 167.

7. Metzler, R.; Klafter, J. Phys. Rep. 2000, 339, 1.

8. Balakrishnan, V. Physica A 1985, 132, 569.

9. Barkai, E.; Metzler, R.; Klafter, J. Phys. Rev. E 2000, 61, 132.

10. Schneider, W. R.; Wyss, W. J. Math. Phys. 1989, 30, 134.

11. Havlin, S.; ben-Avraham, D. Adv. Phys. 1987, 36, 695.

12. ben-Avraham, D.; Havlin, S. Diffusion and Reactions in Fractals and Disordered Systems; Cambridge University Press: Cambridge, 2000.

13. The Fractal Approach to Heterogeneous Chemistry; Avnir, D., Ed.; Wiley: New York, 1989.

14. Blumen, A.; Klafter, J.; White, B. S.; Zumofen, G. Phys. Rev. Lett. 1984, 53, 1301.

15. O'Shaughnessy, B.; Procaccia, I. Phys. Rev. Lett. 1985, 54, 455; Phys. Rev. A 1985, 32, 3073

16. Metzler, R.; Glöckle, W. G.; Nonnenmacher, T. F. Physica A 1994, $211,13$.

17. Metzler, R.; Nonnenmacher, T. F. J. Phys. A: Math. Gen. 1997, 30, 1089.

18. Campos, D.; Méndez, V.; Fort, J. Phys. Rev. E 2004, 69, 031115.

19. Klafter, J.; Zumofen, G.; Blumen, A. J. Phys. A: Math. Gen. 1991, $24,4835$.

20. Jakobs, A.; Kehr, K. W. Phys. Rev. B 1993, 48, 8780.

21. Abramowitz, M.; Stegun, I. A. Handbook of Mathematical Functions; Dover: New York, 1972.

22. Seki, K.; Wojcik, M.; Tachiya, M. J. Chem. Phys. 2003, 119, 7525.

23. Press, W. H.; Flannery, B. P.; Teukolsky, A. A.; Vetterling, W. T. Numerical Recipes; Cambridge University Press: Cambridge, 1986.

24. Srivastava, H. M.; Gupta, K. C.; Goyal, S. P. The H-Functions of One and Two Variables with Applications; South Asian Publishers: New Delhi, 1982. 